\title{
Novel Compact Single Pole Three Throw Switch by using Slow Wave Microstrip Structure
}

\author{
Xinfeng DAI*, Ming ZHOU \\ East Zhongshan Road 524\# \\ Nanjing Electronic Devices Institute, 210016, China \\ *E-mail: dxf_nedi@sina.com \\ $+*$ Corresponding author
}

\begin{abstract}
Slow wave structures have been being widely and deeply investigated up to now, and extensively used in integrated circuits with various applications, such as microwave tubes and miniature components. This paper presents a novel compact single pole three throw (SP3T) switch, and the proposed SP3T involves some sections of novel slow wave microstrip structures, which is constructed by microsrip lines periodically loaded with radial stubs. The periodically loaded microstrip line (PLML) structure is analysed in details. It is revealed that the PLML possesses low insertion loss and good return loss in the pass-band, along with slow wave features, and with good controllable wide bandgap characteristics by tuning its structure parameters. On the basis of the analysis of PLML, the SP3T switch in shunt configuration utilizing PLML structure is designed and manufactured. We obtain good performances and compact size of the SP3T in the experiments. Comparisons of measurements are presented between the conventional and the proposed structures, which verify the validity of this proposed method.
\end{abstract}

Keywords-slow wave structure; periodically loaded microstrip; single pole three throw (sp3t) switch

\section{INTRODUCTION}

Nowadays, the integrated circuits are becoming much smaller with more functions, consequently the compactness of HMICs and MMICs is important and abstractive, which is also helpful to reduce the cost. There are a lot of methods aiming at compact circuit design, and a common sort of appealing method for the compact design is to use slow-wave structures, other than using high permittivity substrate. A large number of literature have discussed the slow wave structures, for instance in [1]-[4]. In [1], a two-dimensional (2-D) photonic bandgap (PBG) structure was utilized to realize slow-wave features, but its slow-wave effects are related to the complicated patterns, including orientation and location of the line with respect to the patterned ground, furthermore this structure is either not convenient for mounting. In [2]-[3], a one-dimensional (1-D) PBG structure CMRC was proposed, but the circuits structure is more complicated. In [4], a uniplanar microstrip periodic structure is developed, unfortunately via holes are utilized in the structure, which are customarily hoped to be avoided.

In this work, a SP3T switch is designed by a novel slow wave structure, which is analysed firstly, and the switch is experimentally demonstrated afterward. The final measurements validate the good performances of the recommended SP3T with smaller size comparing to the conventional structure.

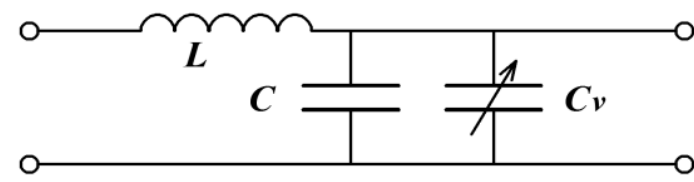

Figure 1. Equivalent lumped circuit model of a NLTL section

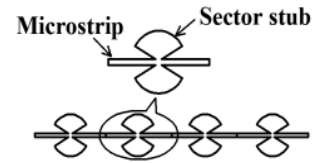

(a)

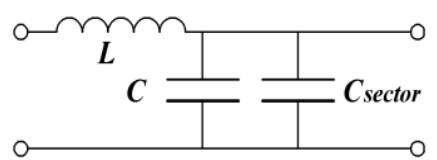

(b)

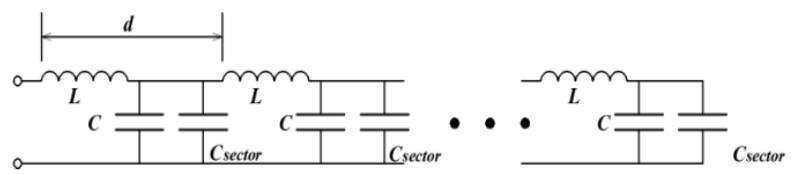

(c)

Figure 2. (a) Microstrip line periodically loaded with radial stubs (b) equivalent circuit of a PLML element (c) equivalent circuit model of the periodically loaded mirostrip line.

\section{DESIGN AND EXPERIMENT OF THE SP3T}

\section{A. Design and Analysis of PLML}

A type of microstrip slow wave structures are usually realized by using uniform transmission line loaded with capacitors, like nonlinear transmission line (NLTL), which has been very hot in recent years, and can be used as sampling circuit driver [5], as well as variable delay time [6]-[8], etc. In a NLTL structure, varactors are always distributed on a coplanar waveguide. Fig 1 shows the equivalent lumped circuit model of a NLTL section. The periodic cutoff (Bragg) frequency of a NLTL circuit is:

$$
f_{\text {Bragg }}=\frac{d}{\sqrt{L d\left(C d+C_{v}\right)}}
$$

Where $L, C$ is the equivalent per unit length series inductance and shunt capacitance of the unloaded line, $d$ is 
the periodic length of the cell, $C_{v}$ is the variable capacitance of the varactors. For frequencies much lower than the Bragg cutoff frequency, the group velocity is given by:

$$
V_{g}=\frac{d}{\sqrt{L d\left(C d+V_{V}\right)}}
$$

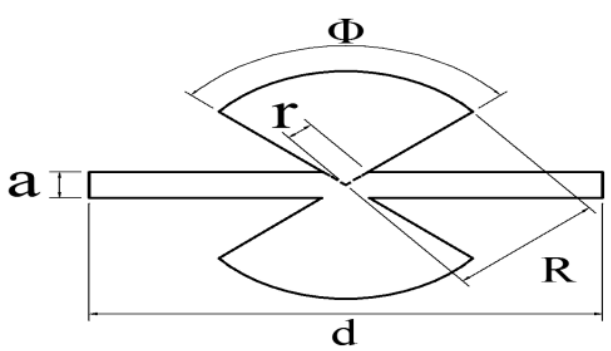

Figure 3. Parameters of a proposed PLML element in details.

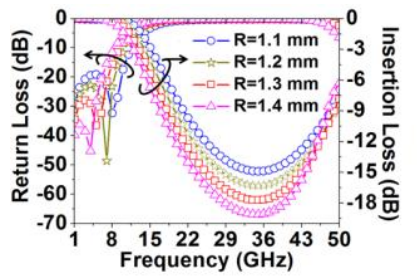

(a)

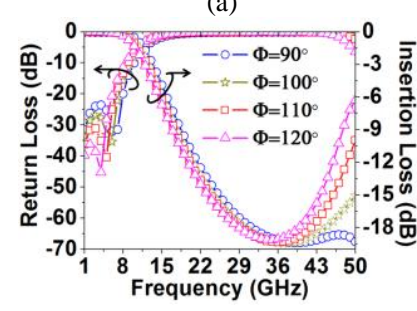

(c)

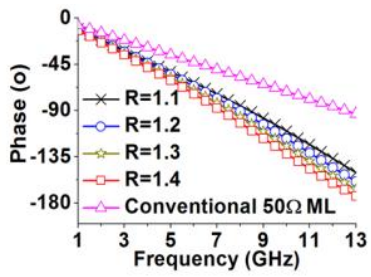

(b)

(d)

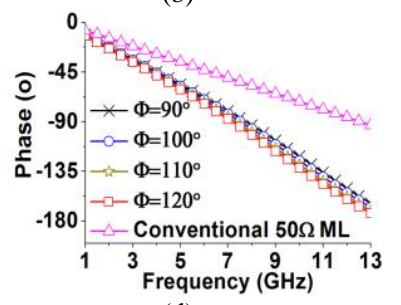

Figure 4. Fig 4. (a) S-parameters and (b) phase responses of a PLML element with different $\mathrm{R}$ of the radial stub. (c) S-parameters and (d) phase responses of a PLML element with different $\Phi$ of the radial stub.

A novel design has been adopted in [10], as shown in Fig 2(a). The microstrip is periodically loaded with radial stubs, which has been used as lumped capacitive stub in the microwave circuit design [9]. Fig 2 (b) and (c) show the equivalent circuit of a circuit element and of the whole periodically loaded mirostrip line.

The characteristic impedance of an unloaded transmission line is given by:

$$
Z_{0}=\sqrt{\frac{L}{C}}
$$

For the proposed stub-loaded microstrip structure, the characteristic impedance ${ }^{Z}$ is determined by:

$$
Z_{L}=\sqrt{\frac{L d}{C d+C_{s t u b}}}
$$

Obviously, in order to make characteristic impedance of the loaded microstrip line to match to $50 \Omega,{ }^{Z_{0}}$ must be set higher than $50 \Omega$.

\section{B. Design and experiment of the SP3T}

Afterwards, a copper clad laminate Rogers/Duroid $5880\left(\varepsilon_{r}=2.2\right)$ with $0.254 \mathrm{~mm}$ thickness is chosen to make a design, and the IE3D software is used to simulate the circuit. Fig 3 shows the configuration parameters of the element in details. By tuning the width and length of the microstrip line, as well as the stub size, the PLML element port impedance can be matched to $50 \Omega$ very well. A section of microstrip loaded with radial stubs is taken as an example, with $\mathrm{a}=0.25 \mathrm{~mm}$ and

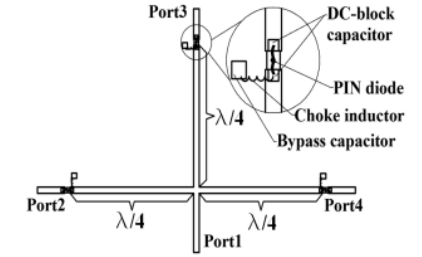

(a)

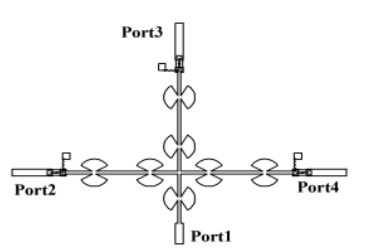

(b)
Figure 5. (a) Conventional microstrip shunt SP3T switch configuration. (b) Proposed compact shunt SP3T switch configuration.

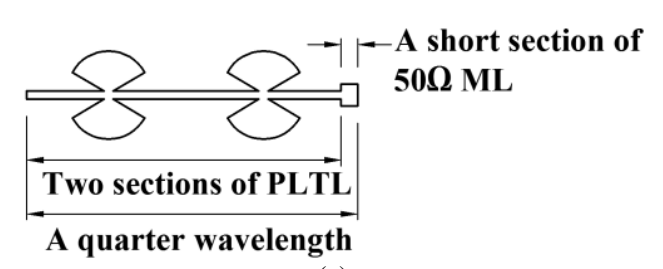

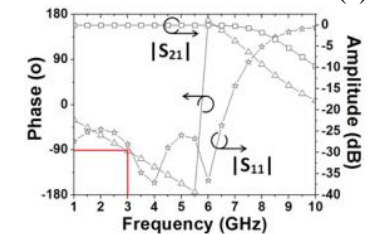

(b)

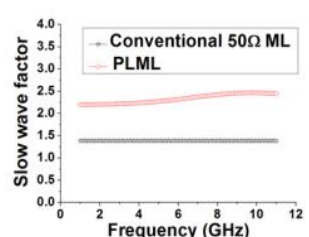

(c)
Figure 6. (a) Equivalent quarter wavelength PLML structures. (b) Simulated S-parameters of the proposed structure. (c) Simulated slow wave factor of the proposed structure.

$\mathrm{d}=4.346 \mathrm{~mm}$, whose original characteristic impedance is about $96.5 \Omega$, and $\square=120^{\circ}, \mathrm{r}=0.2 \mathrm{~mm}$, subsequently, the stub radial $\mathrm{R}$ is respectively set as $1.1,1.2,1.3$ and $1.4 \mathrm{~mm}$. Fig 4 shows the simulated results of the PLML element. In Fig 4(a), the 3dB cutoff frequency is respectively about $15.2 \mathrm{GHz}, 14 \mathrm{GHz}, 12.9 \mathrm{GHz}$ and $11.9 \mathrm{GHz}$, and the bandgap is wide due to the wide-band resonance feature of the radial stub structure, and it can be controlled by tuning the radial of the radial stub. In addition, Fig 4(b) shows the phase responses of the PLML element with different stub radials comparing with that of a section of $50 \Omega$ characteristic impedance microstrip line (ML) of the same physical length. According to the curves, the phase responses of the PLML element are retarded in comparison to the conventional $50-\Omega \mathrm{ML}$, namely, the signal phase velocity is reduced while propagating along the PLML. Its 
slow-wave effects are apparent especially when the stub radial is increased.

Fig 4(c)-(d) present the response of the PLML element with constant $\mathrm{R}=1.4 \mathrm{~mm}$, and the angle of the radial stub is respectively set as $120^{\circ}, 110^{\circ}, 100^{\circ}$ and $90^{\circ}$. The insertion point between the radial stub and the microstrip is kept the same as the case of $\square=120^{\circ}$ and $r=0.2 \mathrm{~mm}$ by changing $\mathrm{r}$. Likewise, the bandgap characteristics and slow wave effects are observed that the bandgap depth is more sensitive to $\mathrm{R}$ than to, and so are the phase responses.

Subsequently, a single pole three throw switch in shunt configuration is designed by the proposed structure to prove its availability. Generally speaking, for single-pole multi-throw switches, the switching devices, for instance diodes, are mounted in series, shunt, or series-shunt configurations across

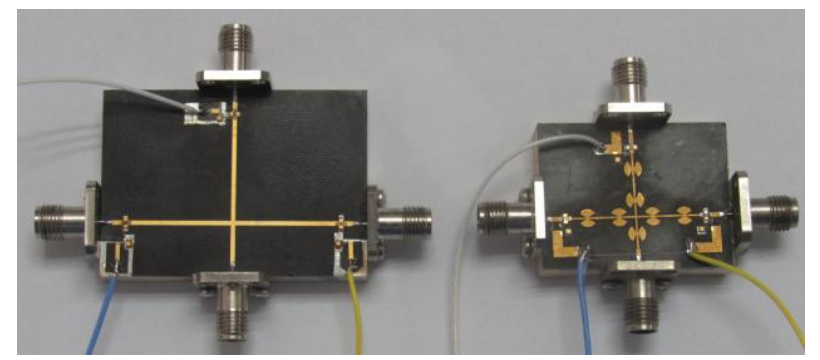

Figure 7. Photograph of the conventional and the proposed SP3T.

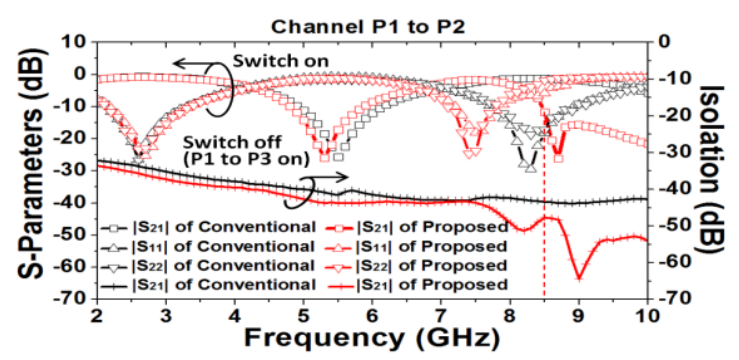

(a)

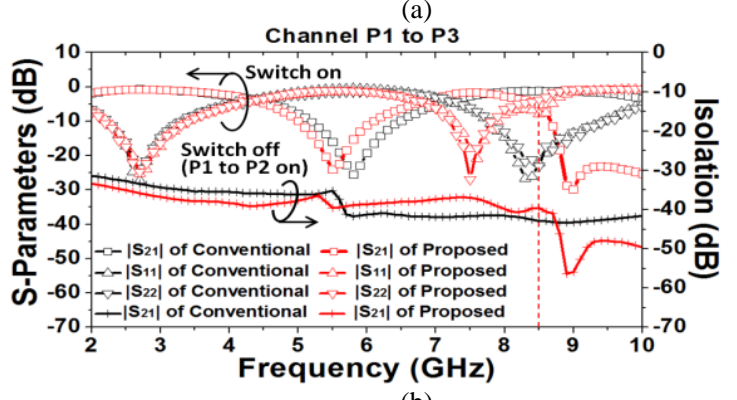

(b)

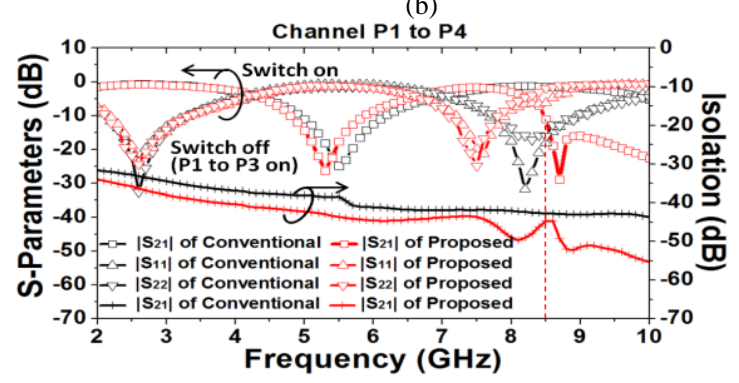

(c)

Figure 8. Comparisons of the measured results of the conventional and the proposed shunt configuration SP3T. the transmission line [11]. The conventional microstrip SP3T shunt switch is composed of three sections of ML, and three shunt diodes, as shown in Fig 5(a). But for such a strcuture, the circuit size becomes large due to the lines, especially for low frequencies. The proposed PLML structure is selected to design the SP3T switch in a classic shunt configuration, as shown in Fig 5(b). Two sections of PLML and a short section of 50- $\Omega$ ML are utilized to replace the conventional quarter wavelength $\mathrm{ML}$ with proper phase response, as shown in Fig 6(a). The center operation frequency of the SP3T is set at $3 \mathrm{GHz}$. The above-mentioned Rogers/Duroid 5880 circuit board is also adopted for the circuit design. Simulated results of the selective structure are shown in Fig 6(b) and (c). The insertion phase at $3 \mathrm{GHz}$ is $91.83^{\circ}$. The short section of $50-\Omega$ ML is also purposely used as a pad for mounting a dc-block capacitor for its wider line-width. And the total physical length is $11.7128 \mathrm{~mm}$, but at the same frequency, the conventional $\lambda / 450 \Omega \mathrm{ML}$ is about $18.46 \mathrm{~mm}$ long, thus $36.55 \%$ length is reduced. The bandgap is also shown in Fig 6(b) when the frequency is over about $8.5 \mathrm{GHz}$, and the slow wave factor (SWF) is indicated in Fig 6(c).

In practice, three MA4AGSW1 PIN diode switches are respectively mounted in the three throw arms by sold paste. The DC-blocking function is fulfilled by the SKYWORKS chip capacitor (SC04701518) of $47 \mathrm{pF}$, and the choke inductors about $12 \mathrm{nH}$ are realized by the coil of gold wires. Besides, the by-pass capacitors are $100 \mathrm{pF}$ chip capacitors (SC10002430).

The photograph of the conventional and the proposed SP3T are shown in Fig 7. The former is distinctly bigger than the latter. Measuring has been carried out, the bias current of each pin diode is set as $11 \mathrm{~mA}$, and the comparisons are also made between the proposed and the conventional in Fig 8. The measurements show that the novel SP3T works well, and the performance is very close to the conventional scheme, whose insertion loss is about $1 \mathrm{~dB}$ from $2.2 \sim 3.2 \mathrm{GHz}$, with return loss less than $-11 \mathrm{~dB}$. There is a frequency shift of about $300 \mathrm{MHz}$ from the designed center frequency $3 \mathrm{GHz}$, which is deemed to be caused mainly by some practical factors, such as material permittivity error and fabrication errors. In the Fig 8 , it is observed that the s-parameters of the conventional and the proposed do not coincide with each other when the throw arm's electrical length is close and more than half wavelength, especially when the pass band is close to the PLML's bandgap, which is due to the slow wave effects of the proposed structure as shown in Fig 6(c). And the bandgap features of the proposed structure are clearly presented in $\left|S_{11}\right|$ of the states of switching on and off beyond $8.5 \mathrm{GHz}$, and it is rational according to the simulated results of the PLML structure in Fig 6(b).

\section{CONCLUSION}

A novel PLML structure is presented and analyzed. It is shown that the PLML structure has low insertion loss and good return loss characteristics in the pass band, as well as slow-wave features. Moreover, the proposed PLML structure has a wide controllable bandgap which can be controlled by tuning its structure parameters. Finally, a novel shunt configuration SP3T switch is designed and 
manufactured by using the PLML structure, good performances are achieved with miniature size superior to the conventional case. This circuit structure is a promising candidate for miniaturized microwave and millimeter-wave circuits.

\section{REFERENCES}

[1] Fei-Ran Yang, et al, A novel Low-Loss Slow-Wave Microstrip Structure. IEEE Microw. Guided Wave Lett. vol.8, no. 11 (1998) 372-374.

[2] Q. Xue, K. M. Shum, and C. H. Chan. Novel 1-D Microstrip PBG cells. IEEE Microw. Guided Wave Lett., vol. 10 (2000) 403-405.

[3] Kam Man Shum, Quan Xue, and Chi Hou Chan. A Novel Microstrip Ring Hybrid Incorporating a PBG Cell. IEEE Microw. Wireless Compon. Lett., vol.11, no. 6 (2001) 258-260.

[4] W. S. Chang and C.Y. Chang. Uniplanar microstrip periodic structure for microstrip circuits. Electron. Letters., vol. 47, No. 8 (2011) 499-500.

[5] Mark J. W. Rodwell, Masayuki Kamegawa, et al. GaAs nonlinear transmission lines for picosecond pulse generation and millimeter wave sampling. IEEE Trans. Microw. Theory Tech., vol.39, no.7 (1991) 1194-1204.

[6] R. P. Hsia, W. M. Zhang, C. W. Domier, and N. C. Luhmann Jr.. A hybrid nonlinear delay line-based broad-band phased antenna array. IEEE Microw. Guided Wave Lett., vol.8, no. 5 (1996) 182-184.

[7] W. M. Zhang, R. P. Hsia, C. Liang, G. Song, C. W. Domier, and N. C. Luhmann Jr.. Novel low-loss delay line for broadband phase antenna array applications. IEEE Microw. Guided Wave Lett., vol.6, no. 11 (1996) 395-397.

[8] S. Nagra, and R. A. York. Monolithic GaAs phase shifter with low insertion loss and continuous phase shift at 20GHz. IEEE Microw. Guided Wave Lett., vol.9, no. 1 (1999) 31-33.

[9] H. A. Atwater. Microstrip reactive circuit elements. IEEE Trans. Microw. Theory Tech., vol.31, no.6 (1983) 488-491.

[10] Xinfeng Dai, Ming Zhou. A Novel Periodically Loaded Slow Wave Microstrip Structure. 2016 IEEE International Workshop On Electromagnetics.

[11] Inder Bahl and Prakash Bhartia, Microwave solid state circuit design, 2rd ed. Hoboken, NJ: John Wiley\& Sons, 2003, ch.12, pp.632-648. 